\title{
Dynamic Dominant Index Set for Mobile Peer-to-Peer Networks ${ }^{\star}$
}

\author{
Wei Shi, Shanping Li, Gang Peng, and Xin Lin \\ College of Computer Science, Zhejiang University, \\ Hangzhou, P.R. China 310027 \\ shiwei@zj165.com \\ shan@cs.zju.edu.cn \\ \{e_pglmary, alexlinxin\}@hotmail.com
}

\begin{abstract}
Efficient locating mechanism is the key issue of establishing peer-to-peer (P2P) resource sharing for mobile Ad Hoc networks (MANET). In this paper, a simple concept of Dynamic Dominant Indexing Set (DDIS) is proposed. By periodically advertising information of shared resources on the backbone of current network topology in a distributed fashion, DDIS gains great improvement on user response time, fault tolerance and scalability. Simulations indicate that DDIS simplifies the searching process and greatly reduces the response delay and thus, it is more appropriate for mobile peer-to-peer source sharing especially in emergent situations.
\end{abstract}

\section{Introduction}

Inviting P2P model into mobile ad hoc networks (MP2P) to address problems encountered during the development of mobile ad hoc networks (MANETs) is proved to be a promising method. Like $\mathrm{P} 2 \mathrm{P}$ computing, one of the major challenges of MP2P computing is resource locating and routing. In order to improve users' experience, mechanisms are seriously required to quickly locate the desired information.

To address resources locating problem in MP2P, we should take following factors into account: 1) in order to lookup the resources more accurately, locating mechanism should guarantee the freshness of search results; 2) many scenarios in MP2P environment, for example emergency rescue and combat, require searching response time as short as possible; 3 ) being aware of the entire network topology is a mission impossible for mobile devices; and 4) individual peer's fault will not cause the crash of the whole system.

Establishing a dynamic lookup set should be an efficient and feasible approach in MP2P networks. In this paper, we propose a locating mechanism in MP2P

\footnotetext{
* This paper is supported by National Natural Science Foundation of China (No. 60473052) and Zhejiang Provincial Natural Science Foundation of China (No.602032).
} 
environment called Dynamic Dominant Index Set (DDIS). DDIS dynamically constructs the index set which is based on the Minimum Connected Dominating Set (MCDS). MCDS is the minimum set of nodes such that every node in the network is either in the set or is a neighbor of a node in the set. According to the information of shared resources periodically published on MCDS, peers can locate the resources quickly by consulting a limited number of peers rather than bothering more other peers. However, finding the MCDS of a specific network has been proved to be a NP-complete problem [1]. We improved the Dominating Pruning (DP) algorithm that proximately solves the MCDS problem in a distributed fashion. With the help of the improved DP algorithm, the DDIS mechanism can build the MCDS-based index set of current MP2P network topology in a more efficient way.

The paper is organized as follows. In the next section, the related work is discussed. Section 3 introduces our searching mechanism. Simulation results and analyzes are presented in section 4 . Finally we conclude our study in section 5 .

\section{Related Work}

In 2] the author put forward a locating mechanism in MP2P network, named PDI. PDI gains considerably good performance. The Dominating Pruning (DP) algorithm [1], computes the approximation of MDCS in a distributed manner according to 2-hop neighborhood information. But DP algorithm only concentrates on constructing an efficient routing algorithm by reducing the broadcast redundancy in MANET and does not take into account the MP2P-specific factors. DDIS improves DP to make it more suitable for building an effective search index in MP2P environment.

\section{The DDIS Mechanism}

Here, the unit disk graph [3] $G=(V, E)$, is introduced to represent a MP2P network, where $V$ represents a set of mobile peers and $E$ represents a set of edges. We use $N(u)$ to represent the neighbor set of $u$. $N(N(u))$ represents the neighbor set of $N(u)$. And we assume that $u$ (sender) and $v$ (receiver) are neighbors.

In our scenario, when a peer launches or forwards an advertising packet, it would decide the forward list around itself. The forward list in the advertising packet would notify his neighbors who belong to the index set and should take the responsibility to cache the metadata in the advertising packet. Meanwhile, by analyzing the forward list in the advertising packet, the receivers can calculate their own forward lists in a more accurate fashion, which will reduce the broadcast redundancy.

Peer willing to share his resources periodically launches a "ADV" broadcast containing the peer's id, the information of his shared resource, the expiring time of shared resource, and the forward list of that packet. Due to the nature 
of broadcasting in MANET, all neighbors around the source peer will get the packet and only the peers in the forward list of that packet are suggested to cache and forward the packet. Others can just simply discard it. Then the receivers calculate their own forward lists and rebroadcast the packet from the source peer. This process continues until the whole network is covered. The forward list of $v$ in DDIS can be computed in the following way, note that $F(u)$ here denotes as the forward list of $u$ passed to $v$ :

1. Let $F(u, v)=[]$ (empty list), $Z=\phi$ (empty set) and $K=\cup S_{i}$ where $S_{i}=N\left(v_{i}\right) \cap(U(u, v)-N(N(v) \cap F(u))), S_{i}=N\left(v_{i} \cap P(u, v)\right)$ for $v_{i} \in B(u, v)$.

2. If there exists any peer $w$ in $P$ that $v$ can notify only through $v_{n}, F(u, v)=$ $F(u, v) \| v_{n}, Z=Z \cup S_{n}, K=K-S_{n}$, and $S_{j}=S_{j}-S_{n}$ for all $S_{j} \in K$. This step repeats until no peer in $P$ that $v$ can notify only through $v_{n}$.

3. Find set $S_{k}$ with the maximum size in $K$. (In case of a tie, the one with the smallest identification $k$ is selected.)

4. $F(u)=F(u, v) \| v_{k}, Z=Z \cup S_{k}, K=K-S_{k}$, and $S_{j}=S_{j}-S_{k}$ for all $S_{j} \in K$.

5. If no new node is added to $Z$, exit; otherwise, goto step 3 .

Here $B(u, v)=N(v)-N(u)$. DP algorithm evaluates the number of peers in $U(u, v)=N(N(v))-(N(u) \cup N(v))$, so called "evaluating peers", which can be covered by $v_{i}$ in decision of including/excluding $v_{i}$ in/from $F(u, v)$. However, the evaluating peers can be even fewer because the peers in the forward list of $u$ will notify the ones in $N(N(v) \cap F(u))$. So these peers can be safely excluded from $U(u, v)$ in DDIS and the evaluating peers can be denoted as $P(u, v)=$ $U(u, v)-N(N(v) \cap F(u))$, recall that $U(u, v)$ represents the uncovered peers 2 hops away from $v$ in DP algorithm.

In selection process of building forward list, DDIS firstly picks up the neighbors that will consequentially be included in $v$ 's forward list because without them $v$ can not inform all the peers in $P(u, v)$. In DP algorithm, these peers, what we call "key neighbors" in this paper, will also be inevitably included in forward list. But since DP algorithm is a kind of greedy algorithm and the selection sequence is random, there may exist unnecessary peers in resulting forward list whose coverage is a part of union of key peers' neighbors. By choosing key neighbors firstly, the forward list of DDIS can be smaller than the one that DP algorithm builds.

With the help of the improved DP algorithm, DDIS can deliver the metadata of shared resources on the dominant index set of current network topology more efficiently. Peers in DDIS frequently check whether the cached metadata is expired. Querying peer firstly checks his cached data to see whether there is information of the required resources or, otherwise, just asks his neighbors to find where the required resources are.

\section{Performance Results}

In this section, we present the simulation results of our DDIS mechanism. We used an IEEE 802.11 standard MAC layer and a standard physical layer de- 


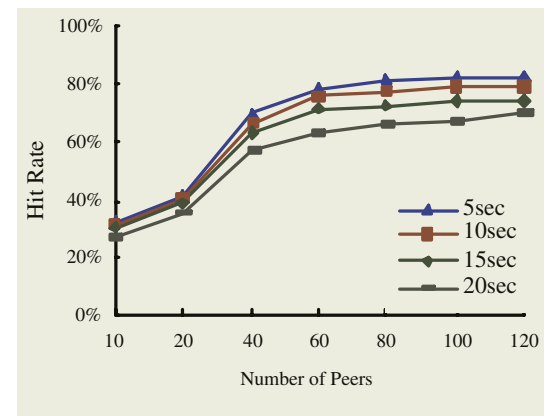

Fig. 1. Hit rate of DDIS with different advertising intervals

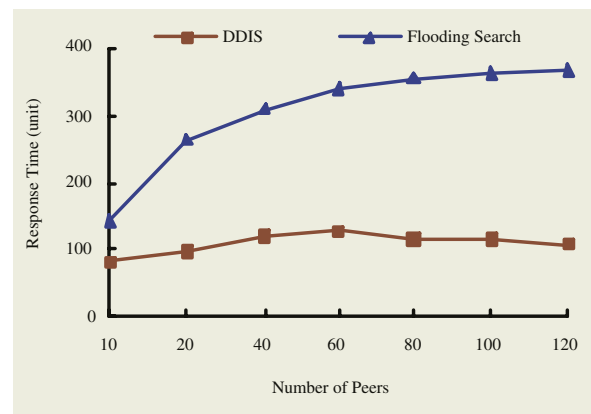

Fig. 2. Response time of the two mechanisms

ploying two-ray ground propagation as radio propagation model. $N_{\text {Peers }}$ peers roaming in an area of $1000 \mathrm{~m} \times 1000 \mathrm{~m}$. Fig. 1 illustrates the hit rate of DDIS with different advertising intervals. As we can see, the shorter advertising interval we set, the higher hit rate we get. However, the performance improvement is achieved at the expense of heavier overhead. Balancing the accuracy of queries and the overhead DDIS must pay needs more efforts of study. From Fig. 2, the main advantage of DDIS can be learned. By dynamically caching the metadata of the peers on the backbone of current network, DDIS gains great improvement on user response time compared with flooding search mechanism. Our goal is to build a small index set to affect all the peers in the network. By putting the metadata around the participants, they can quickly locate their favorable resources without more efforts. Since the required information is put closely to the requiring peers, the response time of DDIS changes little when the number of participants grows. The flooding search mechanism, on the contrary, by broadcasting in the network to locate the target peer which may be far away from the querying peer takes about average 2 to 3 times longer than DDIS to response the users' queries.

\section{Conclusions and Future Work}

In this paper, we put forward a novel concept of DDIS. In fact, DDIS is a kind of replication of index center. By selecting peers in MDCS, the advertisements are delivered close to every peer, which is especially valuable in emergent situations where response time is much more the key issue.

\section{References}

1. H. Lim and C. Kim: Flooding in wireless ad hoc networks. Computer Communications Journal, 24(3-4): 353-363, 2001. 
2. C. Lindemann and O. Waldhorst: A Distributed Search Service for Peer-to-Peer File Sharing in Mobile Applications. Proc. The 2nd International Conference on Peer-to-Peer Computing, September 2002.

3. B.N. Clark, C.J. Colbourn, and D.S. Johnson: Unit Disk Graphs. Discrete Math, vol. 86 , pp. 165-177, 1990. 